\title{
Loss of LKB1-NUAK1 Signalling Enhances NF-KB Activity in a Spheroid Model of High-Grade Serous Ovarian Cancer
}

\section{Adrian Buensuceso \\ Western University \\ Jamie Lee Fritz \\ Western University}

Olga Collins

London Regional Cancer Program

\section{Yudith Ramos Valdes}

London Regional Cancer Program

\section{Gabriel E. DiMattia}

Western University

Trevor G. Shepherd ( $\nabla$ tshephe6@uwo.ca )

Western University

\section{Research Article}

Keywords: high-grade serous ovarian cancer, spheroid, metastasis, LKB1, NUAK1, NF-KB, reactive oxygen species

Posted Date: October 6th, 2021

DOl: https://doi.org/10.21203/rs.3.rs-951554/v1

License: (c) (i) This work is licensed under a Creative Commons Attribution 4.0 International License.

Read Full License

Version of Record: A version of this preprint was published at Scientific Reports on February 22nd, 2022. See the published version at https://doi.org/10.1038/s41598-022-06796-2. 


\section{Abstract}

High-grade serous ovarian cancer (HGSOC) is an aggressive malignancy often diagnosed at an advanced stage. Although most HGSOC patients respond initially to debulking surgery combined with cytotoxic chemotherapy, many ultimately relapse with platinum-resistant disease. Thus, improving outcomes requires new ways of limiting metastasis and eradicating residual disease. We identified previously that Liver kinase B1 (LKB1) and its substrate NUAK1 are implicated in EOC spheroid cell viability and are required for efficient metastasis in orthotopic mouse models. Here, we sought to identify additional signalling pathways altered in EOC cells due to LKB1 or NUAK1 loss-of-function. Transcriptome analysis revealed that inflammatory signalling mediated by NF-KB transcription factors is hyperactive due to LKB1-NUAK1 loss in HGSOC cells and spheroids. Upregulated NF-KB signalling due to NUAK1 loss suppresses reactive oxygen species (ROS) production and sustains cell survival in spheroids. NF-KB signalling is also activated in HGSOC precursor fallopian tube secretory epithelial cell spheroids, and is further enhanced by NUAK1 loss. Finally, immunohistochemical analysis of OVCAR8 xenograft tumors lacking NUAK1 displayed increased RelB expression and nuclear staining. Our results support the idea that NUAK1 and NF-KB signalling pathways together regulate ROS and inflammatory signalling, supporting cell survival during each step of HGSOC pathogenesis. We propose that their combined inhibition may be efficacious as a novel therapeutic strategy for advanced HGSOC.

\section{Introduction}

Epithelial ovarian cancer (EOC) is the most common gynaecologic malignancy in the developed world and is typically diagnosed after it has already metastasized. Consequently, fewer than $30 \%$ of patients survive longer than five years after diagnosis ${ }^{1}$. While the majority of advanced-stage EOC patients initially respond to cytoreductive surgery and combination chemotherapy, most will ultimately relapse with chemo-resistant disease ${ }^{2}$. Therefore, increased understanding of the molecular and cellular adaptations supporting the survival and growth of EOC cells during metastasis may yield novel approaches to improve patient outcomes.

Liver kinase B1 (LKB1; encoded by STK11 in humans) is a serine-threonine kinase that is expressed in most cell types and is recognized as a master regulator of metabolism ${ }^{3}$. LKB1 and its direct target substrate AMP-activated protein kinase (AMPK) comprise an integral signalling axis that senses depletion of intracellular ATP levels, enabling restoration of energy homeostasis and permitting survival during metabolic stress ${ }^{4}$. While LKB1 has tumour-suppressive function in several disease contexts ${ }^{5-11}$, a growing body of evidence supports the notion that it can also promote cancer metastasis ${ }^{12-15}$. Although AMPK is the most well-characterized substrate of LKB1, our recent data indicate that LKB1 acts independently of AMPK to elicit pro-survival mechanisms in EOC metastasis ${ }^{16}$. There are several other known LKB1 substrates ${ }^{17}$, such as the AMPK-related kinases (ARKs), which consist of brain-specific kinases (BRSKs), involved in promoting neuronal polarity ${ }^{18}$, salt-inducible kinases (SIKs), involved in regulating cell polarity, proliferation, apoptosis, and lipid metabolism ${ }^{19}$, the nua kinases (NUAKs), which 
regulate adhesion ${ }^{20}$, motility ${ }^{21}$, proliferation ${ }^{22}$ and stress responses ${ }^{23}$, the microtubule affinity-regulating kinases (MARKs), which regulate cell polarity and motility ${ }^{24}$, and sucrose non-fermenting related kinase (SNRK), which is expressed primarily in testes and may regulate spermatogenesis ${ }^{25}$. Through a proteomic screen followed by direct functional analysis, we determined that LKB1 regulates NUAK1 expression and phosphorylation in EOC cells, and that LKB1-NUAK1 activity is required for metastasis using in vitro and in vivo models of the disease ${ }^{16,20}$, consistent with a previous report linking NUAK1 with poor prognosis in ovarian cancer ${ }^{26}$.

LKB1 has been implicated in inflammatory pathway regulation in multiple contexts ${ }^{27,28}$, including cancer ${ }^{29}$. Inflammation evolved as a protective response to trauma, becoming activated when an organism is subjected to injury, toxicity, or infection ${ }^{30}$. Commonly, this response acts to recruit immune cells to the site of damage to neutralize pathogens while simultaneously regulating tissue repair pathways ${ }^{31}$. Nuclear factor kappa-light-chain-enhancer of activated B cells (NF-KB) signalling is wellestablished as a key proinflammatory regulator mediated by two different pathways ${ }^{32}$. The canonical pathway has perhaps been best characterized in the context of inflammation and is typically activated by numerous proinflammatory cytokines, which act through cell surface receptors to activate the IKB kinase complex via transforming growth factor b-activated kinase 1 (TAK1) ${ }^{33,34}$ and its associated proteins, leading to nuclear translocation of NF-KB transcription factors and target gene regulation. In contrast, non-canonical NF-KB pathway activation is mediated by NF-KB-inducing kinase $(\mathrm{NIK})^{35}$, which regulates the proteolytic processing of NF-KB subunit p100 into its active p52 form ${ }^{36,37}$, leading to dimerization with RelB to affect NF-KB target gene expression. In addition to activation by proinflammatory cytokines, $\mathrm{NF}-\mathrm{kB}$ signalling may become activated in response to various cellular stresses, such as oxidative stress $^{38}$, ionizing radiation ${ }^{39}$, and ultraviolet light ${ }^{40}$.

LKB1 activity has been demonstrated as having a repressive effect on NF-KB signalling $27,28,41$, while the role of NUAK1 in regulating NF-KB proinflammatory signalling is unknown. However, NUAK1 is required in colorectal cancer cells for oxidative stress-induced nuclear factor erythroid 2-related factor (NRF2) activation ${ }^{23}$, which can suppress proinflammatory cytokine gene expression ${ }^{42}$. As such, it is plausible that NUAK1 has context-specific effects on inflammatory signalling. Herein, we show that genetic ablation of LKB1 or NUAK1 in EOC cells is associated with overactive NF-KB signalling, and is further exacerbated in multicellular clusters, or spheroids. However, we demonstrate that there are differences between LKB1 and NUAK1 in utilization of NF-KB signalling to elicit gene expression changes. Notably, elevated NF-KB transcription factor nuclear localization and inflammatory gene expression is correlated with decreased reactive oxygen species (ROS) levels and increased cell viability in spheroids lacking NUAK1, effects that are negated by treatment with the IkB kinase (IKK) inhibitor BAY 11-7082.

\section{Results}

\section{Inflammation-associated transcriptional signatures in EOC cells lacking LKB1 or NUAK1}


We previously reported that knockout of LKB1 in EOC cells results in decreased metastatic potential both in vitro and in vivo ${ }^{16,43}$, while NUAK1 ablation was more limited to altered cell adhesion and reduced tumour growth ${ }^{20}$. To interrogate additional molecular mechanisms underlying these phenotypes due to LKB1 and NUAK1 loss, we performed microarray-based transcriptome and subsequent pathway analysis. Gene set enrichment analysis ${ }^{44,45}$ revealed enrichment of the HALLMARK_TNFA_SIGNALING_VIA_NFKB signature in EOC cells lacking LKB1 or NUAK1 as compared with control cells in both adherent and spheroid culture conditions (Figure 1A, B). Interestingly, this gene set was also enriched in spheroids as compared with adherent cells for both OVCAR8 and iOvCa147 cell lines (Supplementary Figure S2A). We validated our microarray results by RT-qPCR in which specific NF-kB target genes were significantly increased in OVCAR8-STK11KO or OVCAR8-NUAK1KO cells and spheroids (Figure 1C, D). Expression of these genes was assessed in iOvCa147-STK11KO and HeyA8-STK11KO cells, but their upregulation was observed to a lesser degree for some targets (Figure 1E, F), consistent with the NF-KB pathway's ability to selectively activate subsets of target genes in different contexts ${ }^{46}$. Thus, these results indicate that NF-KB transcriptional activity may be enhanced due to the ablation of either LKB1 or NUAK1 activity in EOC cells, particularly in the context of spheroids.

\section{NF-KB signalling is activated due to LKB1-NUAK1 loss in EOC cells}

Given our transcript-level data indicating enhanced NF-KB target gene expression in EOC cells lacking LKB1 or NUAK1, we directly evaluated NF-KB pathway activation. Canonical NF-KB signalling regulates gene expression primarily via heterodimers of RelA (also referred to as p65) and p50. Transcriptional activity of NF-KB complexes is controlled by a combination of IKB-regulated nuclear localization and various post-translational modifications ${ }^{47}$. For example, phosphorylation of RelA at S536 is associated with its increased transcriptional activity ${ }^{47,48}$. Phospho-RelA (S536) is significantly increased in parental EOC cell line spheroids (Figure 2A and Supplementary Figure S2E), consistent with our results indicating an enhanced NF-KB transcriptional signature in spheroids (Supplementary Figure S2A-D). Under adherent conditions, OVCAR8-STK11KO cells exhibit significantly increased p-RelA (S536) compared to OVCAR8 control cells, but this was not further increased in spheroids (Figure 2B). In contrast, we observed that $\mathrm{p}$ RelA (S536) was significantly increased in OVCAR8-NUAK1KO spheroid cells as compared with OVCAR8 spheroids, but there was no significant difference observed in adherent cells (Figure 2B).

These results indicate that knockout of STK11 or NUAK1 significantly increases phosphorylation of RelA at serine 536, which may explain the enhanced expression of NF-KB target genes in EOC spheroids due to LKB1/NUAK1 loss. Next, we sought to assess nuclear abundance of several NF-KB family transcription factors: RelA and c-Rel, which mediate gene expression via the canonical NF-KB pathway, and RelB and p52, which mediate gene expression via the non-canonical pathway.

Transcription factors of the NF-KB family reside predominantly in the cytoplasm due to interactions with inhibitory subunits to keep them in an inactive state ${ }^{49}$. Upon pathway activation, NF-KB transcription factors are released from inhibition and translocate to the nucleus to activate specific target gene expression. Therefore, to further assess NF-KB pathway activation, we evaluated transcription factor 
abundance in cytoplasmic and nuclear fractions. Nuclear abundance of RelA, RelB, and c-Rel was significantly elevated in OVCAR8 spheroids lacking NUAK1 as compared with parental cell spheroids, whereas c-Rel alone was increased in OVCAR8-STK11KO spheroids (Figure 3A, B). Proteolytic processing of $\mathrm{p} 100$ to $\mathrm{p} 52$ is required for non-canonical NF-KB signalling activation ${ }^{50}$. We observed a significant increase in p52 whole-cell abundance in both OVCAR8-STK11KO and OVCAR8-NUAK1KO spheroids as compared with controls (Supplementary Figure S3A, B). These results indicate that canonical and noncanonical NF-KB signalling are elevated due to LKB1 or NUAK1 loss in EOC spheroids, but levels of each transcription factor may be different in each context. No significant differences were seen for RelA, RelB, or c-Rel nuclear localization in native OVCAR8 spheroids as compared with adherent cells (Figure 3A, B), suggesting that enhanced NF-KB target gene expression in OVCAR8 spheroids may be the result of RelA (S536) phosphorylation.

\section{Activated NF-KB signalling promotes spheroid cell viability in the context of NUAK1 loss.}

LKB1 and its direct substrate NUAK1 have been implicated in mediating cellular adaptation to stress $^{4,23,51}$. Therefore, ablation of either of these kinases in EOC cells may necessitate additional stress responses to maintain cell viability. Given our results, as well as evidence by others that NF-KB signalling is activated as an adaptive response due to LKB1 loss $27,28,41$, we sought to ablate NF-KB inflammatory signalling in our system. BAY 11-7082 is a small-molecule inhibitor against IKK1 and IKK2 ${ }^{52,53}$, which are required for non-canonical and canonical NF-KB pathways, respectively ${ }^{54}$. We assessed nuclear abundance of RelA and RelB, as they represent primary transcription factors for canonical and noncanonical NF-KB signalling, respectively. Indeed, BAY 11-7082 treatment (Supplementary Figure S4) decreased RelA and RelB nuclear abundance in OVCAR8, OVCAR8-STK11KO and OVCAR8-NUAK1KO spheroids (Figure 4A). BAY 11-7082 also substantially decreased p-RelA (S536) in OVCAR8-NUAK1KO spheroids, with more modest decreases observed in OVCAR8 and OVCAR8-STK11KO spheroids (Figure 4A). In addition, we confirmed that BAY 11-7082 treatment blocked the upregulation of several NF-KB target genes in our experimental model: i.e., KLF9 in OVCAR8 and OVCAR8-STK11KO spheroids, OLR1 in OVCAR8-STK11KO spheroids, and IL1B in both adherent and spheroid NUAK1KO cells (Figure 4B).

To address our idea that increased NF-KB signalling promotes stress-induced cell survival in EOC spheroids, we assessed the effect of NF-KB blockade on EOC spheroid viability. Intriguingly, BAY 11-7082 significantly decreased viable cell number in OVCAR8-NUAK1KO spheroids compared to DMSO-treated controls, but this effect was not observed in either OVCAR8 or OVCAR8-STK11KO spheroids (Figure 4C, D). NUAK1 may be required for protection from oxidative stress as observed in colorectal cancer cells ${ }^{23}$, and NF-KB-mediated antioxidant gene expression occurs in EOC cells ${ }^{55}$. Thus, increased NF-KB signalling in OVCAR8-NUAK1KO cells may be required to abrogate elevated oxidative stress due to ROS. Indeed, OVCAR8-NUAK1KO spheroids had significantly reduced ROS levels as compared with OVCAR8 spheroids, but this was reversed when treated with BAY 11-7082, leading to decreased cell viability (Figure 4E-H). Since the importance of NF-KB signalling activity appeared to be crucial for EOC cell viability in the context of NUAK1 loss, we focused our subsequent analysis using NUAK1KO cells. 
Given that nuclear abundance for both RelA and RelB is significantly elevated in OVCAR8-NUAK1KO spheroids cells (Figure 3A, B), we sought to determine whether knockout of NUAK1 in EOC cells specifically necessitates canonical or non-canonical NF-KB signalling to promote spheroid cell viability. We performed transient siRNA-mediated knockdown of RELA and RELB (Supplementary Figure S5), which are the primary mediators of transcription in the canonical- and non-canonical NF-kB pathways, respectively. Knockdown was confirmed by immunoblot analysis in lysates prior to seeding cells for spheroids, and when spheroid cell viability was assessed after seven days (Supplementary Figure S5A). Decreased NF-KB activity was confirmed by assessing IL $1 B$ and $I L 1 A$ transcription, two NF-KB target genes with observed upregulation in OVCAR8-NUAK1KO cells (Supplementary Figure S5B).

Knockdown of RELA and RELB, alone or in combination, did not decrease OVCAR8-NUAK7KO spheroid cell viability (Supplementary Figure S5C), indicating that additional IKK- and/or NF-KB-associated signalling mechanisms may be involved in promoting OVCAR8-NUAK1KO spheroid cell viability.

\section{NUAK1 loss increases NF-KB signalling in fallopian tube epithelial cells}

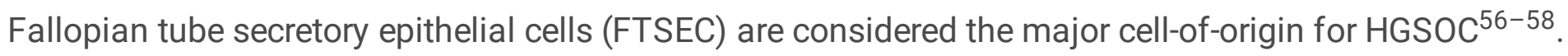
To determine whether increased NF-KB signalling due to NUAK1 ablation is specific to HGSOC cells or may also be a property of pre-neoplastic FTSEC cells, we performed CRISPR/Cas9-mediated knockout of NUAK1 in FT190 cells, an immortalized human fallopian tube secretory epithelial cell line ${ }^{59}$. We observed a significant increase in RelA nuclear abundance in FT190-NUAK1KO, but not parental FT190, spheroids as compared with adherent cells (Figure 5A, B). RelB nuclear abundance was elevated in spheroids as compared with adherent cells for both FT190-NUAK1KO cells and FT190 controls (Figure 5A, B). Moreover, a trend towards increased nuclear abundance of RelA in FT190-NUAK1KO spheroids as compared with FT190 control spheroids was observed ( $p=0.0545$; Figure 5B). Further to this, there was a significant increase in expression of two representative NF-KB target genes, IL $1 A$ and IL 1B, in FT190NUAK1KO adherent cells as compared with FT190 controls (Figure 5C). These differences were not statistically significant in spheroids, however, perhaps due to the already elevated nuclear abundance of NF-KB transcription factors in both FT190 and FT190-NUAK1KO spheroid cells (Figure 5A, B). These results suggest that NUAK1 loss has the capacity to enhance NF-KB signalling even in precursor cells of HGSOC.

\section{Elevated nuclear RelB expression in OVCAR8-NUAK1KO xenograft tumours}

Spheroids are key contributors to EOC metastasis via characteristics that favour dissemination, such as evasion of anoikis ${ }^{60}$ and resistance to chemotherapy, and they have transcriptional profiles that differ from primary and secondary adherent tumours ${ }^{61}$. To determine whether enhanced NF-KB signalling due to NUAK1 loss is a spheroid-specific property or is present in solid tumours as well, we assessed nuclear localization of RelA and RelB by immunohistochemical staining in mouse intraperitoneal tumour xenografts generated as part of a previous study ${ }^{20}$. We observed significantly increased positive nuclear staining for RelB in OVCAR8-NUAK1KO xenograft tumours as compared with OVCAR8 tumours, however 
no significant differences in RelA staining were observed (Figure 6A-C). These results indicate that activated NF-KB signalling due to NUAK1 loss in spheroids is sustained in metastatic tumour deposits, but the relative contribution of canonical and non-canonical NF-KB signalling may differ in each context during spread of disease.

\section{Discussion}

We previously reported that depletion of LKB1 in EOC cells decreases metastatic potential in suspension culture and orthotopic mouse xenograft models of metastasis ${ }^{16,43}$. Furthermore, our recent work on the direct LKB1 substrate NUAK1 revealed key roles in regulating adhesion molecules and promoting metastatic potential in $\mathrm{EOC}^{20}$. In the current study, we present intriguing new evidence that NF-KB signalling is activated in EOC spheroid cells which is further enhanced by ablation of LKB1-NUAK1 function. Interestingly, there may be differences between LKB1 and NUAK1 requirements for NF-KB signalling regulation of either canonical or non-canonical pathways. Lastly, specific loss of NUAK1 increases ROS levels and necessitates enhanced NF-KB signalling for the maintenance of EOC spheroid cell viability.

EOC spheroids have been widely implicated in promoting spread of disease and supporting the emergence of chemoresistance ${ }^{62}$. NF-kB signalling promotes multicellular tumour spheroid formation and growth in multiple cancer cell line models ${ }^{63}$. For example, RelA promotes cell proliferation while RelB supports viability of tumour-initiating cells, and both act to promote spheroid formation ${ }^{64}$. Herein, we also show enhancement of an NF-KB transcriptional signature in EOC spheroids with increased p-RelA (S536) in these structures. Proinflammatory NF-KB signalling in EOC is typically associated with poor patient outcomes, owing to the protection it confers from chemotherapy and immune surveillance. Furthermore, NF-KB signalling is recognized as an important stress response pathway in EOC, protecting against oxidative stress, anoikis, and other stressors ${ }^{55,65,66}$. As such, enhanced NF-KB signalling in spheroids may be an adaptation that promotes metastasis in EOC. Spheroids, considered a primary vehicle for EOC cell transit during metastasis, may contribute to elevated proinflammatory cytokines found in ascites associated with immunosuppression.

Our previous studies have implicated a crucial role for intact LKB1-NUAK1 signalling in promoting spheroid formation, cell viability, and metastatic potential ${ }^{16,20,43}$. Further investigation of the altered transcriptome in EOC spheroids lacking either LKB1 or NUAK1 led to our discovery of enhanced NF-KB signalling pathway activation. Several other reports support the idea that LKB1 can repress NF-KB signalling. Depletion of LKB1 is associated with enhanced NF-KB signalling in B cells ${ }^{41}$ and skeletal muscle ${ }^{28}$. LKB1 can interact with IKK $\beta$ leading to repression of NF-KB signalling in macrophages ${ }^{27}$. There are fewer reports demonstrating cross-talk regulation between NUAK1 and NF-KB signalling. NUAK1 may activate the canonical NF-KB pathway via phospho-RelA in the lung cancer cell line A54967. However, our results demonstrate NUAK1 loss enhances NF-KB signalling in the high-grade serous cell line OVCAR8, as well as in the precursor cell model FT190. It is well-established that NF-KB signalling is activated by 
numerous cellular stressors ${ }^{46}$ and, amongst its many functions, can promote cell survival ${ }^{68}$. Given that NUAK1 may serve as an important response to metabolic ${ }^{69}$ and oxidative stress ${ }^{23}$, NUAK1 ablation may subject spheroid cells to increased levels of these particular stresses. Indeed, we observed that OVCAR8NUAK1KO spheroid cells require enhanced NF-KB signalling to deal with ROS and maintain spheroid cell viability. Further to these findings, we observed that immunohistochemical staining for nuclear RelB, but not RelA, was elevated in xenografts of OVCAR8-NUAK1KO cells, indicating sustained activation of noncanonical NF-KB signalling in metastatic implants. Taken together, our findings suggest that loss of NUAK1 necessitates inflammatory stress response signalling in early precursor lesions through to latestage metastasis during a broad window of disease progression.

In many cell types, the oxidative stress response is primarily mediated by the NRF2 pathway, which drives expression of antioxidant genes ${ }^{70}$. In colorectal cancer cells, NUAK 1 is activated by oxidative stress and is required for nuclear import of NRF2 and subsequent induction of antioxidant response genes ${ }^{23}$. Perhaps, in our system, loss of NUAK1 forces EOC spheroid cells to adopt NF-KB mediated mechanisms to respond to elevated oxidative stress as an alternative pathway. We observed that KLF9 expression is significantly decreased while expression of $I L 1 B$ and several other pro-inflammatory cytokines are significantly increased due to NUAK1 loss. These results would suggest impaired NRF2 signalling since ROS-induced expression of $K L F 9$ is known to occur via the NRF2 pathway ${ }^{71}$, while expression of $I L 1 B$ and other pro-inflammatory cytokines is repressed by NRF2 ${ }^{42}$. Indeed, treatment of OVCAR8-NUAK1KO spheroids with the IKK inhibitor BAY 11-7082 increased ROS levels, which is consistent with a recent report that NF-kB-associated expression of antioxidant genes protects ovarian cancer cells from oxidative stress $^{55}$. Thus, enhancement of NF-KB signalling may be an adaptive response to impaired ROS clearance in NUAK1-deleted spheroid cells.

While ROS play important roles in cell signalling, excessive levels clearly damage macromolecules critical to cell survival and induce multiple types of cell death. As such, ROS induction has been considered as a therapeutic strategy for some cancers ${ }^{72}$. If therapeutic strategies were to be employed targeting NUAK1 in advanced EOC, particularly within the harsh environment of malignant ascites ${ }^{73-75}$, perhaps combination with antioxidant inhibitors would be required for maximum efficacy. Our findings warrant further investigation to determine whether targeting NUAK1 can enhance the effectiveness of therapeutic strategies that benefit from ROS induction in cancer cells.

\section{Methods}

\section{Antibodies and Reagents:}

Antibodies against p-RelA-S536 (\#3033; 1:1000), RelA (\#8242; 1:1000), RelB (\#4922, 1:2000; IHC: \#10544), c-Rel (\#4727; 1:1000), NF-KB2 (\#3017; 1:1000), LKB1 (\#3050; 1:1000), and NUAK1 (\#4458; 1:1000) were purchased from Cell Signalling Technology (Danvers, MA). Antibodies against lamin $A / C$ (MAB3211; 1:500) and actin (A2066; 1:20000) was purchased from Millipore (Temecula, CA, USA). 
Antibody against tubulin (T5168; 1:20000) was purchased from Sigma. HRP-conjugated antibodies against mouse IgG (NA931; 1:10000) and rabbit IgG (NA934; $1: 10000)$ were purchased from Cytiva. All antibodies were diluted in tris-buffered saline-Tween 20 containing $5 \%$ bovine serum albumin, with the exception of NUAK1 (tris-buffered saline-Tween 20 containing 5\% nonfat milk). BAY 11-7082 was purchased from Cayman Chemical (cat. 10010266; Ann Arbor, MI, USA). ROS-Glo $\mathrm{H}_{2} \mathrm{O}_{2}$ Assay was purchased from Promega (cat. G8820).

\section{Generation of STK11KO and NUAK1KO cell lines:}

Generation of OVCAR8-STK11KO ${ }^{16}$ and OVCAR8-NUAK1KO ${ }^{20}$ cell lines has been previously described. For FT190-NUAK1KO cells, two independent 20-nucleotide guide sequences targeting the NUAK1 gene 5'GTGGC GGGGG ACCGC CCCGA-3' (site 1) and 5'-GGGTC TCCTG CAGCT CGTAG CGG-3' (site 2) were selected using the CRISPR Design Tool (http://tools.genome-engineering.org). Complementary oligonucleotides 5'-CACCG TCGGG GCGGT CCCCC GCCAC-3' and 5'-CACCG GGGTC TCCTG CAGCT CGTAG-3' for site 1 and 5'-CACCG GGGTC TCCTG CAGCT CGTAG-3' and 5'-AAACC TACGA GCTGC AGGAG ACCCC-3' for site 2 (Sigma-Genosys) were annealed and ligated into the Bbsl-digested restriction endonuclease site of pSpCas9(BB)-2A-Puro plasmid ${ }^{76}$ (gift from Dr. F. Dick, Western University) to generate the pSpCas9-sgNUAK1-1 and -2 plasmids. Cells were seeded at 200,000 cells/well into 6-well plates and transfected with $0.5 \mu \mathrm{g}$ each of pSpCas9-sgNUAK1 plasmids using LipofectAMINE2000 (Invitrogen) according to the manufacturer's instructions. Media containing $1 \mu \mathrm{g} / \mathrm{mL}$ puromycin was replaced the following day, and cells were treated for one day. After growth recovery, the cells were trypsinized, counted, and seeded into 96 -well plates to perform limiting dilution subcloning of NUAK1knockout cells. Single colonies were expanded for protein isolation and the confirmation of NUAK1 loss by immunoblotting. Four clones lacking NUAK1 protein expression were identified by immunoblotting and pooled to generate a mixed population.

For experiments in Figures 1-3, 4A-D, 6, and Supplementary Figures S1A, S3, S4A-B, OVCAR8-NUAK1KO clone 62 was used. For experiments in Figures 4E-H and Supplementary Figures S1C, S4C, and S5, a mixed population containing equal proportions of 3 OVCAR8-NUAK1KO clones $(50,62,63)$ were used to confirm that results were not clone-specific.

\section{Cultured cell lines:}

OVCAR8, OVCAR8-STK11KO, OVCAR8-NUAK1KO, HeyA8, and HeyA8-STK11KO cell lines were cultured in RPMI-1640 (Wisent). iOvCa147, iOvCa147-STK11KO, FT190, and FT190-NUAK1KO cell lines were cultured in DMEM/F12 (Life Technologies). For all cell lines, growth medium was supplemented with $10 \%$ fetal bovine serum. OVCAR8 and HeyA8 cells were obtained from the American Type Culture Collection; iOvCa147 cells were generated by our research group as previously described ${ }^{77}$. Adherent cells were maintained on tissue culture-treated polystyrene (Sarstedt, Newton, NC, USA). Spheroids were maintained in Ultra-Low Attachment (ULA) cluster plates (Corning, NY, USA). The immortalized human fallopian tube secretory epithelial cell line FT $190^{59}$ was provided by R. Drapkin (University of Pennsylvania, 
Philadelphia, PA). All cell lines were authenticated by short tandem repeat analysis performed by The Centre for Applied Genomics (The Hospital for Sick Children, Toronto, ON, Canada) and routinely tested for mycoplasma using the Universal Mycoplasma Detection Kit (30-1012K; ATCC).

\section{Microarray Analysis:}

Cells were seeded into $10 \mathrm{~cm}$ tissue culture-treated dishes $\left(1 \times 10^{6}\right.$ cells in $12 \mathrm{~mL}$; 1 dish per sample) or 6 well ULA plates $\left(5 \times 10^{5}\right.$ cells in $5 \mathrm{~mL}$; 3 wells per sample). Spheroids were harvested for RNA purification 24h post-seeding; adherent cells were harvested 48h post-seeding. RNA was collected as described in RNA collection and purification, and two $100 \mathrm{ng} / \mu \mathrm{L}$ dilutions in nuclease-free water were prepared for each sample: $3 \mu \mathrm{L}$ for Bioanalyzer analysis to confirm acceptable RNA prep quality, and $10 \mu \mathrm{L}$ for Clariom $S$ microarray analysis. Dilutions were frozen on dry ice and shipped to The Centre for Applied Genomics (The Hospital for Sick Children, Toronto, ON, Canada) for further processing. Data analysis and export was performed using the Transcriptome Analysis Console 4.0.1 software package (Thermo Fisher).

\section{RNA collection and purification:}

RNA was extracted using the RNEasy Spin Column kit (cat. 74104; Qiagen) according to the manufacturer's protocol, with the optional DNasel (cat. 79254; Qiagen) treatment. Adherent cells were collected by aspirating medium and scraping cells into $600 \mu \mathrm{L}$ Buffer RLT and stored at $-80^{\circ} \mathrm{C}$ until processing. Spheroids were pelleted at $800 \mathrm{~g}, 4^{\circ} \mathrm{C}$, medium was aspirated, and cells were lysed in $350 \mu \mathrm{L}$ Buffer RLT and stored at $-80^{\circ} \mathrm{C}$ until processing. RNA concentration, $A_{260 / 280}$, and $A_{280 / 230}$ were determined using a NanoDrop One Microvolume UV-Vis Spectrophotometer (Thermo Scientific).

\section{Gene Set Enrichment Analysis:}

Sample signals were exported using the Transcriptome Analysis Console 4.0.1 software package and formatted according to GSEA guidelines (https://software.broadinstitute.org/cancer/software/gsea/wiki/index.php/Data_formats) for Text file format for expression dataset. Phenotype labels were generated according to the Categorical class file format. The following GSEA (ver. 3.0) parameters were used: gene sets database,

“h.all.v6.2.symbols.gmt"; permutation type, "gene_set”; number of permutations, 1000; collapse/remap to gene symbols, collapse; chip platform, “Clariom_S_Human.r1.chip”, enrichment statistic, weighted; metric for ranking genes, Signal2Noise; gene list sorting mode, real; gene list ordering mode, descending; maximum gene set size, 500; minimum gene set size, 15; collapsing mode for probe sets, max_probe; normalization mode, meandiv; randomization mode, no_balance; omit symbols with no match, true; median for class metrics, false; seed for permutation, timestamp. For identifying significantly enriched gene sets, $p$-value and false discovery rate cutoffs of 0.05 and 0.25 , respectively, were used. Venn diagrams in Figure 1A-B and Supplementary Figure S2A were generated using the matplotlib-venn package (https://github.com/konstantint/matplotlib-venn).

\section{Quantitative Reverse Transcription PCR:}


cDNA synthesis was performed using the High Capacity cDNA Reverse Transcription Kit (Thermo Fisher) according to the manufacturer's protocol using $2000 \mathrm{ng}$ RNA per reaction, yielding a final volume of $20 \mathrm{uL}$ per reaction. Reactions were incubated in a MyCycler thermocycler (BioRad) using the following settings: $25^{\circ} \mathrm{C}$ for $10 \mathrm{~min}, 37^{\circ} \mathrm{C}$ for $120 \mathrm{~min}, 8^{\circ} \mathrm{C}$ for $5 \mathrm{~min}$, hold at $4^{\circ} \mathrm{C}$ until sample retrieval. Each reaction was then diluted with an equal volume of nuclease-free water. qPCR was performed using the Brilliant II SYBR Green QPCR Master Mix (Agilent Technologies) according to the manufacturer's protocol scaled to a 10 $\mu \mathrm{L}$ reaction volume. Reactions were assembled in a 96-well plate, sealed with adhesive plastic film, and then centrifuged for $20 \mathrm{~s}$ to remove bubbles. Cycling was performed in a QuantStudio 3 RT-PCR System (Thermo Fisher) using built-in settings for SYBR Green Chemistry, Fast mode. Data analysis was performed using QuantStudio Design and Analysis Software 1.4.3. Fold-change values relative to controls were calculated using using the $2^{-\Delta \Delta C}{ }_{T}$ method ${ }^{78}$. Primer sequences (Supplementary Table S2) were obtained from https://www.origene.com and purchased from Invitrogen.

\section{Preparation of whole-cell, cytoplasmic and nuclear lysates:}

For assessment of total p-RelA (S536), day 3 whole-cell lysates were generated from adherent cells cultured at a density of $0.5-3 \times 10^{6}$ cells in $13 \mathrm{~mL}$ medium (10 cm dish), or spheroid cells cultured at a density of $1-3 \times 10^{6}$ cells in $6 \mathrm{~mL}$ medium (35 mm ULA well). For assessment of NF- $\mathrm{KB}$ transcription factor nuclear abundance, day 1 whole, cytoplasmic and nuclear lysates were generated from adherent cells cultured at a density of $3.5-4.5 \times 10^{6}$ cells in $13 \mathrm{~mL}$ medium $(10 \mathrm{~cm}$ dish), or spheroid cells cultured at a density of $1.25-1.5 \times 10^{6}$ cells in $5 \mathrm{~mL}$ medium ( $35 \mathrm{~mm}$ ULA well). Seeding numbers were chosen to obtain acceptable protein yields for each cell line.

Whole-cell lysates: Adherent cells grown in tissue culture-treated plates or dishes were collected by aspirating medium, washing $2 x$ with cold PBS, and scraping into modified RIPA buffer. Spheroids (at least $1.5 \times 10^{6}$ cells per sample) were collected by transferring the cell suspension into a conical tube on ice, pelleting by centrifugation in a swinging bucket rotor $\left(800 \times \mathrm{g}\right.$ at $4^{\circ} \mathrm{C}$ for $\left.4 \mathrm{~min}\right)$, aspirating medium, resuspending in at least $10 \mathrm{~mL}$ cold PBS, pelleting, resuspending again in cold PBS, pelleting, and aspiration of PBS. Cell pellets were then lysed in modified RIPA buffer, vortexed, subjected to one freezethaw cycle, and clarified by centrifugation ( $\max \times \mathrm{g}$ at $4^{\circ} \mathrm{C}$ for $20 \mathrm{~min}$ ).

Cytoplasmic and nuclear lysates: Adherent cells were washed 2x with cold PBS and scraped into cold PBS using a cell lifter, transferred to a $50 \mathrm{~mL}$ conical tube and pelleted by centrifugation in a swinging bucket rotor ( $800 \times \mathrm{g}$ at $4^{\circ} \mathrm{C}$ for $4 \mathrm{~min}$ ). Spheroids (at least $3 \times 10^{6}$ cells per sample) were collected into a conical tube and pelleted by centrifugation in a swinging bucket rotor $\left(800 \times \mathrm{g}\right.$ at $4^{\circ} \mathrm{C}$ for $\left.4 \mathrm{~min}\right)$, washed $2 x$ in cold PBS, and pelleted by centrifugation. Cell pellets were resuspended in at least $450 \mu \mathrm{L}$ cold hypotonic lysis buffer and incubated on ice for $50 \mathrm{~min}$, vortexing (max) for $10 \mathrm{~s}$ every $10 \mathrm{~min}$. The suspension was then pelleted by centrifugation ( $\max \times \mathrm{g}$ at $4^{\circ} \mathrm{C}$ for $3 \mathrm{~min}$ ). The supernatant (cytoplasmic lysate; $90 \%$ of hypotonic lysis buffer volume used) was transferred to another microcentrifuge tube and left on ice. The remaining supernatant in the original microcentrifuge tube was aspirated. $1 \mathrm{~mL}$ of cold wash buffer was added, vortexed for $20 \mathrm{~s}$, pelleted by centrifugation ( $\max \times \mathrm{g}$ at $4^{\circ} \mathrm{C}$ for $1 \mathrm{~min}$ ), and the 
wash buffer was aspirated. This wash step was repeated and the pellet was lysed in $100 \mu \mathrm{L}$ modified RIPA buffer and vortexed to generate the nuclear lysate. Cytoplasmic and nuclear lysates were then subjected to 1 freeze-thaw cycle and clarified by centrifugation ( $\max \times \mathrm{g}$ for $20 \mathrm{~min}$ at $4^{\circ} \mathrm{C}$ ). Hypotonic lysis buffer. 20 mM HEPES (pH 7.4), 1 mM EGTA, 1 mM EDTA, 1 mM DTT, 0.3\% Triton X-100, 1 mM $\mathrm{Na}_{3} \mathrm{VO}_{4}, 10 \mathrm{mM} \mathrm{NaF}, 1 \mathrm{mM}$ PMSF, 1x SIGMAFAST protease inhibitor cocktail (S8820; Sigma), $10 \mathrm{mM}$ beta-glycerophosphate. Fraction Wash buffer. $10 \mathrm{mM} \mathrm{HEPES} \mathrm{(pH} \mathrm{7.4),} 10 \mathrm{mM} \mathrm{KCl,} 0.1 \mathrm{mM}$ EGTA, $0.1 \mathrm{mM}$ EDTA, 1x SIGMAFAST protease inhibitor cocktail. Modified RIPA buffer. $50 \mathrm{mM}$ HEPES (pH 7.4), $150 \mathrm{mM}$ $\mathrm{NaCl}, 10 \%$ glycerol, $1.5 \mathrm{mM} \mathrm{MgCl}_{2}, 1 \mathrm{mM}$ EGTA, $1 \%$ Triton X-100, 0.1\% SDS, $1 \mathrm{mM} \mathrm{Na}_{3} \mathrm{VO}_{4}, 10 \mathrm{mM} \mathrm{NaF}, 1$ mM PMSF, 1x SIGMAFAST protease inhibitor cocktail (cat. S8820; Sigma), $10 \mathrm{mM}$ betaglycerophosphate. Total protein concentration was determined using the Bio-Rad Protein Assay according to manufacturer's instructions (cat. 5000006; Bio-Rad).

\section{Immunoblot Analysis:}

Immunoblotting was performed using the Bio-Rad Mini-PROTEAN II Electrophoresis System (Bio-Rad) according to manufacturer's instructions using gels cast in-house (30\% acrylamide/bis solution 37.5:1, cat. \#1610158; Bio-Rad). Densitometry was performed using the Image Lab 6.0.1 software package (BioRad).

\section{Treatment with BAY 11-7082:}

Cells were seeded into $10 \mathrm{~cm}$ tissue culture-treated dishes at a density of $2.5 \times 10^{6}$ cells in a volume of 12 $\mathrm{mL}$. The following day, cells were pre-treated with drug or vehicle: medium was removed by aspiration and replaced with $10 \mathrm{~mL}$ medium containing $5 \mu \mathrm{M}$ BAY 11-7082 or an equivalent $\mathrm{v} / \mathrm{v}$ DMSO as a vehicle control. The following day, cells were trypsinized, counted and seeded at the required density in medium containing BAY 11-7082 to a final concentration of $5 \mu \mathrm{M}$ or an equivalent v/v DMSO. Seeding densities: 24-well ULA spheroids, $0.5-1 \times 10^{5}$ cells in $1 \mathrm{~mL}$; 6-well ULA spheroids, $0.5-1.5 \times 10^{6}$ cells in $5 \mathrm{~mL}$; 96 -well ULA spheroids, $1 \times 10^{4}$ cells in $80 \mu \mathrm{L} ; 10 \mathrm{~cm}$ adherent, $1.5-2.5 \times 10^{6}$ cells in 10-12 mL. Cell numbers were chosen to achieve acceptable yields.

\section{Trypan blue spheroid viability assay:}

Cells were seeded into 24-well ULA cluster plates $\left(0.5-1 \times 10^{5}\right.$ cells per well in a volume of $\left.1 \mathrm{~mL}\right)$.

Spheroids were collected into microcentrifuge tubes on ice and pelleted by centrifugation at $500 \times \mathrm{g}$ for 3 min. Medium was aspirated and the pellet was washed by resuspension in $500 \mu \mathrm{L}$ PBS (without drawing cells up into pipette tip), pelleted again as described above, resuspended in 50-250 $\mu \mathrm{L}$ trypsin/EDTA, and incubated at $37^{\circ} \mathrm{C}$ with gentle agitation every $10 \mathrm{~min}$ until aggregates were no longer visible (10-30 min). Trypsin was then inactivated by adding an equal volume of FBS. Trypan Blue dye was added (volume equal to trypsin/EDTA + FBS) prior to counting the sample and gently mixed by pipetting. Cell counting was performed using a TC20 Automated Cell Counter (Bio-Rad). 
Spheroids were collected into microcentrifuge tubes on ice using a beveled P200 tip and pelleted at $500 \times$ $\mathrm{g}$ for $5 \mathrm{~min}$ at $4^{\circ} \mathrm{C}$. Medium supernatant was aspirated and the pellet was stored at $-80^{\circ} \mathrm{C}$. Relative viability was assessed by CyQUANT Cell Proliferation Assay (cat. C7026; Thermo Fisher) using 2x CyQUANT GR dye concentration to extend the assay linear detection range as described in the manufacturer's instructions, blank-subtracted, and used to normalize ROS-Glo luminescence.

\section{Measurement of ROS levels in EOC spheroids:}

Cells were pre-treated with BAY 11-7082 or DMSO as described in Treatment with BAY 11-7082. 24h after treatment, cells were trypsinized, counted and seeded into 96-well round-bottom ULA cluster plates at a density of 10000 cells/well in $80 \mu \mathrm{L}$, maintaining the same concentration of DMSO or BAY 11-7082 (5 $\mu \mathrm{M})$. At d7, ROS levels were assessed using the ROS-Glo $\mathrm{H}_{2} \mathrm{O}_{2}$ Assay (Promega) according to the manufacturer's instructions. $20 \mu \mathrm{L}$ of $125 \mu \mathrm{M} \mathrm{H}_{2} \mathrm{O}_{2}$ substrate was added to each well and returned to the cell culture incubator for $5 \mathrm{~h}$. $100 \mu \mathrm{L}$ of complete ROS-Glo detection solution was then added to each well and incubated for $20 \mathrm{~min}$ at room temperature on a rocker. $175 \mu \mathrm{L}$ from each well was then transferred to an opaque white 96-well plate, and luminescence was measured using a Synergy H4 plate reader (Biotek; Winooski, VT, USA). After blank subtraction, ROS-Glo luminescence was normalized to CyQUANT signal measured from identically-seeded and -treated replicate wells.

\section{Xenograft tumour histology and immunohistochemistry:}

Previously archived formalin-fixed, paraffin-embedded tumours ${ }^{20}$ were sectioned at a thickness of $5 \mu \mathrm{m}$. Hematoxylin/eosin staining was used to visualize tissue architecture, and adjacent sections were immunohistochemically stained to assess RelA and RelB (1:200 dilution), using hematoxylin as a counterstain. Sectioning and staining were performed by the Molecular Pathology Core Facility at Robarts Research Institute (London, Ontario, Canada). Images of stained tumour sections were captured using an Aperio ScanScope slide scanner (Leica).

\section{Immunohistochemistry quantification and scoring:}

IHC analysis was performed using the Fiji distribution of Image $\mathrm{J}^{79}$. Images of individual stained tumour nodules were exported using the ImageScope software package (Leica). Empty space around nodules was erased using the Adobe Photoshop software package. Necrotic tumour regions were identified using the "Trainable Weka Segmentation" plugin ${ }^{80}$, confirmed by visual inspection, and cleared. RelA and RelB staining was then evaluated using the "IHC Profiler" plugin ${ }^{81}$ for the total image, and for nuclear regions of the image (identified by thresholding of the hematoxylin channel).

\section{Transient knockdown of RELA and RELB:}

Cells were seeded in 6-well plates $\left(1.5 \times 10^{5}\right.$ cells/well) and transfected using DharmaFECT1 (1/500 final dilution) as per manufacturer's protocol (Dharmacon) using a final total siRNA concentration of $10 \mathrm{nM}$. RELA ON-TARGETplus SMARTpool (L-003533-00-0020), RELB ON-TARGETplus SMARTpool (L-004767- 
00), or ON-TARGETplus Non-targeting Pool (D-001810-10) were used. 24h after transfection, $3 \mathrm{~mL}$ of medium were added to each well, for a total volume of $5 \mathrm{~mL}$. 48 hours later, cells were trypsinized, counted and seeded for experiments. Immunoblot analysis was performed on d0 adherent and d7 spheroid cell lysates to confirm RELA and RELB knockdown. RT-qPCR was performed in d1 spheroid cell and $\mathrm{d} 2$ adherent cell RNA to confirm decreased NF-KB target gene expression.

\section{Microscopy:}

Phase-contrast images were captured using a Leica DMI 4000B inverted microscope. Images of immunohistochemically-stained xenograft tumour sections were captured using an Aperio ScanScope slide scanner (Leica).

\section{Statistical Analysis:}

Statistical analyses were performed using GraphPad Prism 6.05 (GraphPad Software). Specific analysis details are described in figure legends.

\section{Declarations}

\section{ACKNOWLEDGEMENTS}

We thank Dr. Bart Kolendowski for assisting with bioinformatic analysis, Hailey Dall-Proud for RT-qPCR experiments, and Caroline O'Neil for performing IHC at the Robarts Research Institute. This research was supported by funding from the Canadian Institutes for Health Research (Grant No. 136836) to T. Shepherd. We are also very grateful to the many donors to the Mary and John Knight Translational Ovarian Cancer Research Unit through the London Health Sciences Foundation for additional programmatic and infrastructure funding.

\section{AUTHOR CONTRIBUTIONS}

Conception and design: A.B., J.L.F., G.E.D., T.G.S.

Development of methodology: A.B., J.L.F., Y.R.V., T.G.S.

Acquisition of data: A.B., J.L.F., O.C.

Analysis and interpretation of data: A.B., J.L.F.

Writing, review, and/or revision of the manuscript: A.B., T.G.S.

Administrative, technical, or material support: Y.R.V., O.C.

Study supervision: G.E.D, T.G.S. 


\section{References}

1. Siegel, R. L., Miller, K. D. \& Jemal, A. Cancer statistics, 2020. CA. Cancer J. Clin.70, 7-30 (2020).

2. Ushijima, K. Treatment for Recurrent Ovarian Cancer-At First Relapse. J. Oncol.2010, (2010).

3. Shackelford, D. B. \& Shaw, R. J. The LKB1-AMPK pathway: metabolism and growth control in tumour suppression. Nat Rev Cancer9, 563-75 (2009).

4. Hardie, D. G. AMPK--sensing energy while talking to other signaling pathways. Cell Metab20, 939-52 (2014).

5. Guldberg, P. et al. Somatic mutation of the Peutz-Jeghers syndrome gene, LKB1/STK11, in malignant melanoma. Oncogene18, 1777-80 (1999).

6. Sanchez-Cespedes, M. et al. Inactivation of LKB1/STK11 is a common event in adenocarcinomas of the lung. Cancer Res62, 3659-62 (2002).

7. Wingo, S. N. et al. Somatic LKB1 mutations promote cervical cancer progression. PLoS One4, e5137 (2009).

8. Morton, J. P. et al. LKB1 haploinsufficiency cooperates with Kras to promote pancreatic cancer through suppression of p21-dependent growth arrest. Gastroenterology139, 586-97, 597 e1-6 (2010).

9. Gill, R. K. et al. Frequent homozygous deletion of the LKB1/STK11 gene in non-small cell lung cancer. Oncogene30, 3784-91 (2011).

10. Fang, R. et al. Integrative genomic analysis reveals a high frequency of LKB1 genetic alteration in Chinese lung adenocarcinomas. J Thorac Onco/9, 254-8 (2014).

11. George, S. H. et al. Loss of LKB1 and p53 synergizes to alter fallopian tube epithelial phenotype and high-grade serous tumorigenesis. Oncogene35, 59-68 (2016).

12. Trapp, E. K. et al. LKB1 pro-oncogenic activity triggers cell survival in circulating tumor cells. Mol Onco/11, 1508-1526 (2017).

13. Tan, X. et al. Upregulation of liver kinase B1 predicts poor prognosis in hepatocellular carcinoma. Int J Onco/53, 1913-1926 (2018).

14. Charawi, S. et al. LKB1 signaling is activated in CTNNB1-mutated HCC and positively regulates $\beta$ catenin-dependent CTNNB1-mutated HCC. J Patho/247, 435-443 (2019).

15. Zubiete-Franco, I. et al. SUMOylation regulates LKB1 localization and its oncogenic activity in liver cancer. EBioMedicine40, 406-421 (2019).

16. Buensuceso, A., Ramos-Valdes, Y., DiMattia, G. E. \& Shepherd, T. G. AMPK-Independent LKB1 Activity Is Required for Efficient Epithelial Ovarian Cancer Metastasis. Mol Cancer Res18, 488-500 (2020).

17. Lizcano, J. M. et al. LKB1 is a master kinase that activates 13 kinases of the AMPK subfamily, including MARK/PAR-1. EMBO J23, 833-43 (2004).

18. Kishi, M., Pan, Y. A., Crump, J. G. \& Sanes, J. R. Mammalian SAD kinases are required for neuronal polarization. Science307, 929-932 (2005). 
19. Taub, M. Salt Inducible Kinase Signaling Networks: Implications for Acute Kidney Injury and Therapeutic Potential. Int. J. Mol. Sci.20, (2019).

20. Fritz, J. L. et al. A novel role for NUAK1 in promoting ovarian cancer metastasis through regulation of fibronectin production in spheroids. Cancers Base/12, (2020).

21. Legembre, P., Schickel, R., Barnhart, B. C. \& Peter, M. E. Identification of SNF1/AMP kinase-related kinase as an NF-kappaB-regulated anti-apoptotic kinase involved in CD95-induced motility and invasiveness. J. Biol. Chem.279, 46742-46747 (2004).

22. Yuan, W.-C. et al. NUAK2 is a critical YAP target in liver cancer. Nat. Commun.9, 4834 (2018).

23. Port, J. et al. Colorectal Tumors Require NUAK1 for Protection from Oxidative Stress. Cancer Discov8, 632-647 (2018).

24. Marx, A., Nugoor, C., Panneerselvam, S. \& Mandelkow, E. Structure and function of polarity-inducing kinase family MARK/Par-1 within the branch of AMPK/Snf1-related kinases. FASEB J. Off. Publ. Fed. Am. Soc. Exp. Biol.24, 1637-1648 (2010).

25. Jaleel, M. et al. Identification of the sucrose non-fermenting related kinase SNRK, as a novel LKB1 substrate. FEBS Lett.579, 1417-1423 (2005).

26. Phippen, N. T. et al. NUAK1 (ARK5) Is Associated with Poor Prognosis in Ovarian Cancer. Front Onco/6, 213 (2016).

27. Liu, Z. et al. Liver kinase B1 suppresses lipopolysaccharide-induced nuclear factor kappaB (NFkappaB) activation in macrophages. J Biol Chem290, 2312-20 (2015).

28. Chen, T. et al. Liver kinase B1 inhibits the expression of inflammation-related genes postcontraction in skeletal muscle. J Appl Physiol 1985120, 876-88 (2016).

29. Ollila, S. et al. Stromal Lkb1 deficiency leads to gastrointestinal tumorigenesis involving the IL-11JAK/STAT3 pathway. J Clin Invest128, 402-414 (2018).

30. Furman, D. et al. Chronic inflammation in the etiology of disease across the life span. Nat. Med.25, 1822-1832 (2019).

31. Eming, S. A., Wynn, T. A. \& Martin, P. Inflammation and metabolism in tissue repair and regeneration. Science356, 1026-1030 (2017).

32. Taniguchi, K. \& Karin, M. NF-kappaB, inflammation, immunity and cancer: coming of age. Nat Rev Immuno/18, 309-324 (2018).

33. Vidal, S. et al. Mutations in the Drosophila dTAK1 gene reveal a conserved function for MAPKKKs in the control of rel/NF-KB-dependent innate immune responses. Genes Dev.15, 1900-1912 (2001).

34. Silverman, N. et al. Immune Activation of NF-KB and JNK Requires Drosophila TAK1 *. J. Biol. Chem.278, 48928-48934 (2003).

35. Malinin, N. L., Boldin, M. P., Kovalenko, A. V. \& Wallach, D. MAP3K-related kinase involved in NF-KB induction by TNF, CD95 and IL-1. Nature385, 540-544 (1997).

36. Xiao, G., Harhaj, E. W. \& Sun, S.-C. NF-kB-Inducing Kinase Regulates the Processing of NF-kB2 p100. Mol. Cel/, 401-409 (2001). 
37. Senftleben, U. et al. Activation by IKKa of a Second, Evolutionary Conserved, NF-KB Signaling Pathway. Science293, 1495-1499 (2001).

38. Gloire, G., Legrand-Poels, S. \& Piette, J. NF-kappaB activation by reactive oxygen species: fifteen years later. Biochem Pharmacol72, 1493-505 (2006).

39. Brach, M. A. et al. lonizing radiation induces expression and binding activity of the nuclear factor kappa B. J Clin Invest88, 691-5 (1991).

40. Stein, B., Rahmsdorf, H. J., Steffen, A., Litfin, M. \& Herrlich, P. UV-induced DNA damage is an intermediate step in UV-induced expression of human immunodeficiency virus type 1, collagenase, Cfos, and metallothionein. Mol. Cell. Biol.9, 5169-5181 (1989).

41. Walsh, N. C. et al. LKB1 inhibition of NF-kappaB in B cells prevents $T$ follicular helper cell differentiation and germinal center formation. EMBO Rep16, 753-68 (2015).

42. Kobayashi, E. H. et al. Nrf2 suppresses macrophage inflammatory response by blocking proinflammatory cytokine transcription. Nat Commun7, 11624 (2016).

43. Peart, T. et al. Intact LKB1 activity is required for survival of dormant ovarian cancer spheroids. Oncotarget (2015).

44. Subramanian, A. et al. Gene set enrichment analysis: a knowledge-based approach for interpreting genome-wide expression profiles. Proc Natl Acad Sci U A102, 15545-50 (2005).

45. Liberzon, A. et al. The Molecular Signatures Database (MSigDB) hallmark gene set collection. Cell Syst1, 417-425 (2015).

46. Mercurio, F. \& Manning, A. M. NF-kB as a primary regulator of the stress response. Oncogene18, 6163-6171 (1999).

47. Christian, F., Smith, E. L. \& Carmody, R. J. The Regulation of NF-kB Subunits by Phosphorylation. Cells5, (2016).

48. Chen, L.-F. et al. NF-kappaB RelA phosphorylation regulates RelA acetylation. Mol. Cell. Biol.25, 7966-7975 (2005).

49. Oeckinghaus, A. \& Ghosh, S. The NF-kB Family of Transcription Factors and Its Regulation. Cold Spring Harb. Perspect. Biol.1, a000034 (2009).

50. Sun, S.-C. Non-canonical NF-kB signaling pathway. Cell Res.21, 71-85 (2011).

51. Liu, L. et al. Deregulated MYC expression induces dependence upon AMPK-related kinase 5. Nature483, 608-12 (2012).

52. Pierce, J. W. et al. Novel inhibitors of cytokine-induced lkappaBalpha phosphorylation and endothelial cell adhesion molecule expression show anti-inflammatory effects in vivo. $J$ Biol Chem272, 21096-103 (1997).

53. Fernández-Majada, V. et al. Nuclear IKK activity leads to dysregulated notch-dependent gene expression in colorectal cancer. Proc Natl Acad Sci U A104, 276-81 (2007).

54. Israël, A. The IKK Complex, a Central Regulator of NF-KB Activation. Cold Spring Harb. Perspect. Biol.2, a000158 (2010). 
55. Kleinschmidt, E. G. et al. Rgnef promotes ovarian tumor progression and confers protection from oxidative stress. Oncogene38, 6323-6337 (2019).

56. Piek, J. M. et al. Dysplastic changes in prophylactically removed Fallopian tubes of women predisposed to developing ovarian cancer. J Patho/195, 451-6 (2001).

57. Lee, Y. et al. A candidate precursor to serous carcinoma that originates in the distal fallopian tube. $J$ Patho/211, 26-35 (2007).

58. Labidi-Galy, S. I. et al. High grade serous ovarian carcinomas originate in the fallopian tube. Nat Commun8, 1093 (2017).

59. Karst, A. M., Levanon, K. \& Drapkin, R. Modeling high-grade serous ovarian carcinogenesis from the fallopian tube. Proc Natl Acad Sci U A108, 7547-52 (2011).

60. Al Habyan, S., Kalos, C., Szymborski, J. \& McCaffrey, L. Multicellular detachment generates metastatic spheroids during intra-abdominal dissemination in epithelial ovarian cancer. Oncogene (2018) doi:10.1038/s41388-018-0317-x.

61. Ding, Y. et al. Molecular characteristics and tumorigenicity of ascites-derived tumor cells: mitochondrial oxidative phosphorylation as a novel therapy target in ovarian cancer. Mol. Oncol.n/a,

62. Ahmed, N. \& Stenvers, K. L. Getting to know ovarian cancer ascites: opportunities for targeted therapy-based translational research. Front Onco/3, 256 (2013).

63. Gallardo-Pérez, J. C. et al. NF-kappa B is required for the development of tumor spheroids. J. Cell. Biochem.108, 169-180 (2009).

64. House, C. D. et al. NFkappaB Promotes Ovarian Tumorigenesis via Classical Pathways That Support Proliferative Cancer Cells and Alternative Pathways That Support ALDH(+) Cancer Stem-like Cells. Cancer Res77, 6927-6940 (2017).

65. Momeny, M. et al. Blockade of nuclear factor-KB (NF-KB) pathway inhibits growth and induces apoptosis in chemoresistant ovarian carcinoma cells. Int. J. Biochem. Cell Biol.99, 1-9 (2018).

66. Harrington, B. S. \& Annunziata, C. M. NF-KB Signaling in Ovarian Cancer. Cancers11, 1182 (2019).

67. Chen, P., Li, K., Liang, Y., Li, L. \& Zhu, X. High NUAK1 expression correlates with poor prognosis and involved in NSCLC cells migration and invasion. Exp Lung Res39, 9-17 (2013).

68. Piva, R., Belardo, G. \& Santoro, M. G. NF-kappaB: a stress-regulated switch for cell survival. Antioxid. Redox Signal.8, 478-486 (2006).

69. Escalona, E., Muñoz, M., Pincheira, R., Elorza, Á. A. \& Castro, A. F. Cytosolic NUAK1 Enhances ATP Production by Maintaining Proper Glycolysis and Mitochondrial Function in Cancer Cells. Front. Oncol.0, (2020).

70. Nguyen, T., Nioi, P. \& Pickett, C. B. The Nrf2-antioxidant response element signaling pathway and its activation by oxidative stress. J Biol Chem284, 13291-5 (2009).

71. Zucker, S. N. et al. Nrf2 amplifies oxidative stress via induction of Klf9. Mol Cel/53, 916-928 (2014).

72. Zou, Z., Chang, H., Li, H. \& Wang, S. Induction of reactive oxygen species: an emerging approach for cancer therapy. Apoptosis22, 1321-1335 (2017). 
73. Barbolina, M. V. et al. Microenvironmental Regulation of Ovarian Cancer Metastasis. in Ovarian Cancer: Second Edition (eds. Stack, M. S. \& Fishman, D. A.) 319-334 (Springer US, 2010). doi:10.1007/978-0-387-98094-2_15.

74. Kipps, E., Tan, D. S. \& Kaye, S. B. Meeting the challenge of ascites in ovarian cancer: new avenues for therapy and research. Nat Rev Cancer13, 273-82 (2013).

75. Rickard, B. P. et al. Malignant Ascites in Ovarian Cancer: Cellular, Acellular, and Biophysical Determinants of Molecular Characteristics and Therapy Response. Cancers13, 4318 (2021).

76. Ran, F. A. et al. In vivo genome editing using Staphylococcus aureus Cas9. Nature520, 186-191 (2015).

77. Correa, R. J. et al. Combination of AKT inhibition with autophagy blockade effectively reduces ascites-derived ovarian cancer cell viability. Carcinogenesis35, 1951-61 (2014).

78. Livak, K. J. \& Schmittgen, T. D. Analysis of Relative Gene Expression Data Using Real-Time Quantitative PCR and the 2- $\triangle \Delta$ CT Method. Methods25, 402-408 (2001).

79. Schindelin, J. et al. Fiji: an open-source platform for biological-image analysis. Nat Methods $9,676-$ 82 (2012).

80. Arganda-Carreras, I. et al. Trainable Weka Segmentation: a machine learning tool for microscopy pixel classification. Bioinformatics33, 2424-2426 (2017).

81. Varghese, F., Bukhari, A. B., Malhotra, R. \& De, A. IHC Profiler: an open source plugin for the quantitative evaluation and automated scoring of immunohistochemistry images of human tissue samples. PLoS One9, e96801 (2014).

\section{Figures}


A

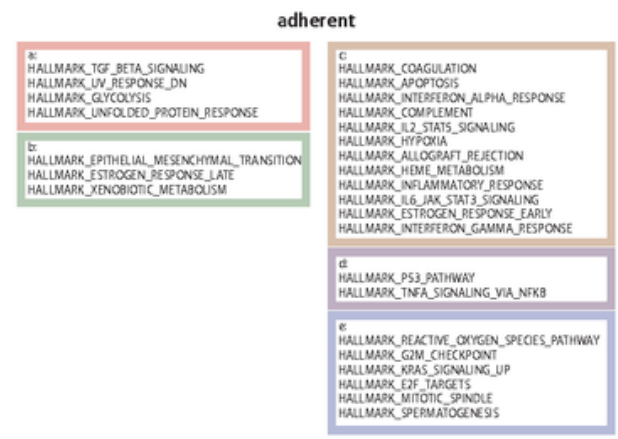

B
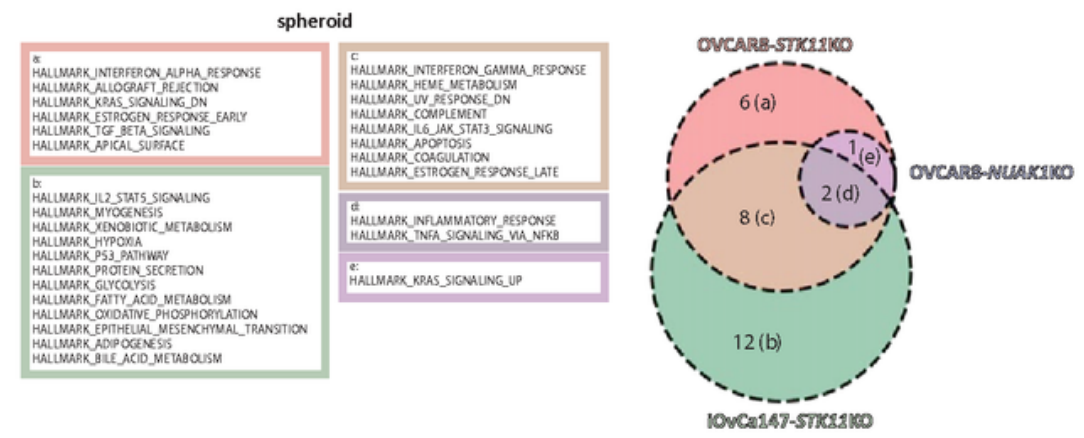

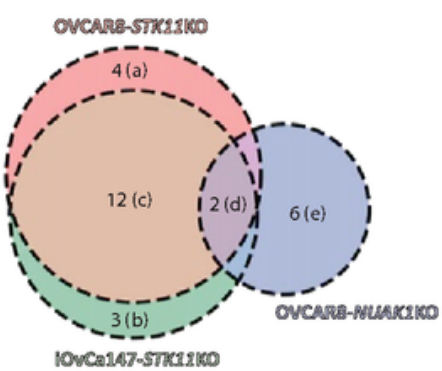

10VCa197-STRerge

10vcars7-smessiec
C

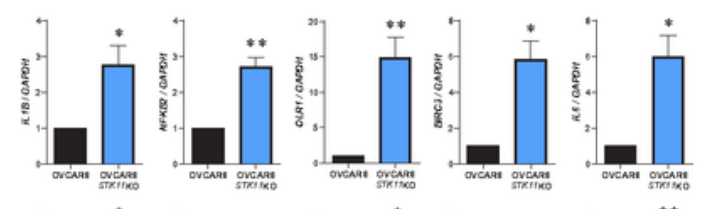

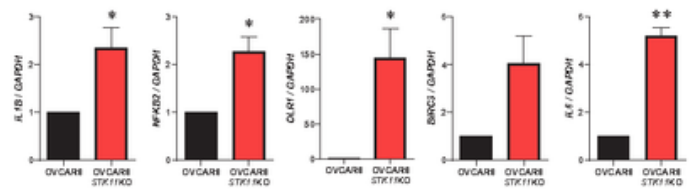

$\mathrm{E}$

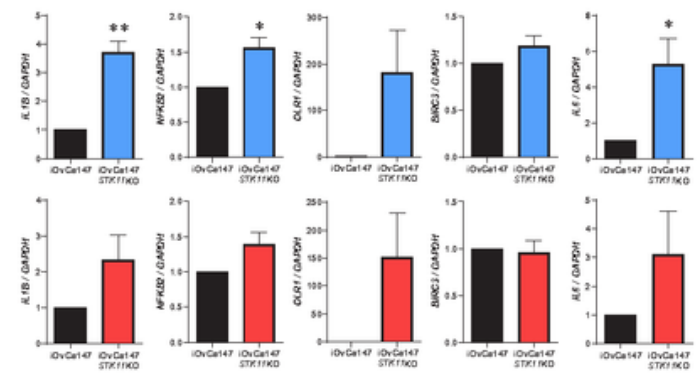

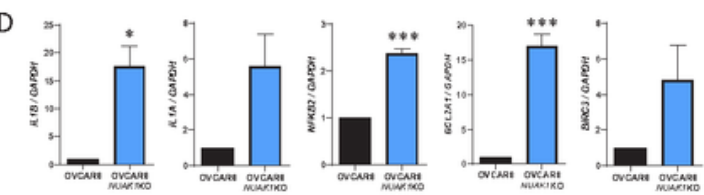

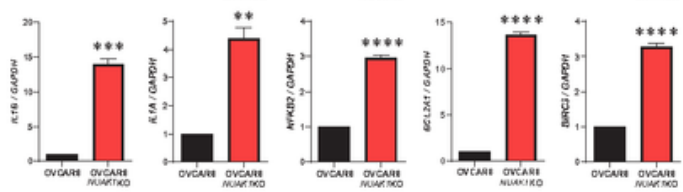

$\mathrm{F}$
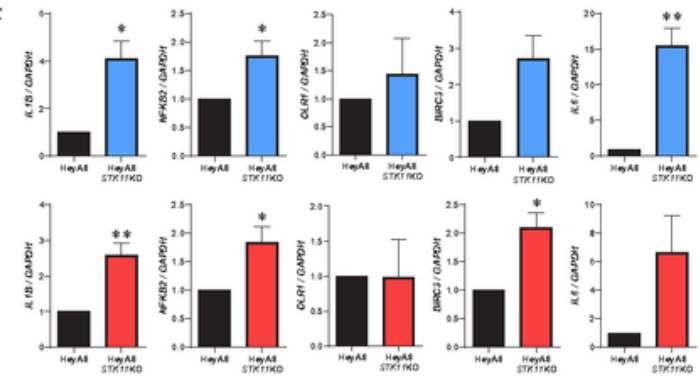

\section{Figure 1}

Enrichment of inflammation-associated transcriptional signatures in EOC cell lines lacking LKB1 or NUAK1. (A, B) Comparison of transcriptional signatures enriched in STK11KO or NUAK1KO cells as compared with parental EOC cells in adherent culture (A) or spheroid culture (B). Lists of Hallmarks were determined using Gene Set Enrichment Analysis (BROAD Institute; p-value cutoff: 0.05, FDR cutoff: 0.25). (C-F) Expression of selected NF-KB-target genes by RT-qPCR in OVCAR8-STK11KO (C), OVCAR8-NUAK1KO 
(D), iOvCa147-STK11KO (E), and HeyA8-STK11KO (F) cell lines. Data from adherent culture of knockout cell lines shown in blue and spheroid culture of knockout cell lines are shown in red. Knockout cells were compared with parental cell controls by unpaired, two-tailed Student's t-test $\left(* p \leq 0.05,{ }^{*} p \leq 0.01\right.$, ${ }^{\star * \star} p$ $\left.\leq 0.001,{ }^{* \star \star *} p \leq 0.0001 ; n=3\right)$. Error bars indicate standard error of the mean (S.E.M.).

A
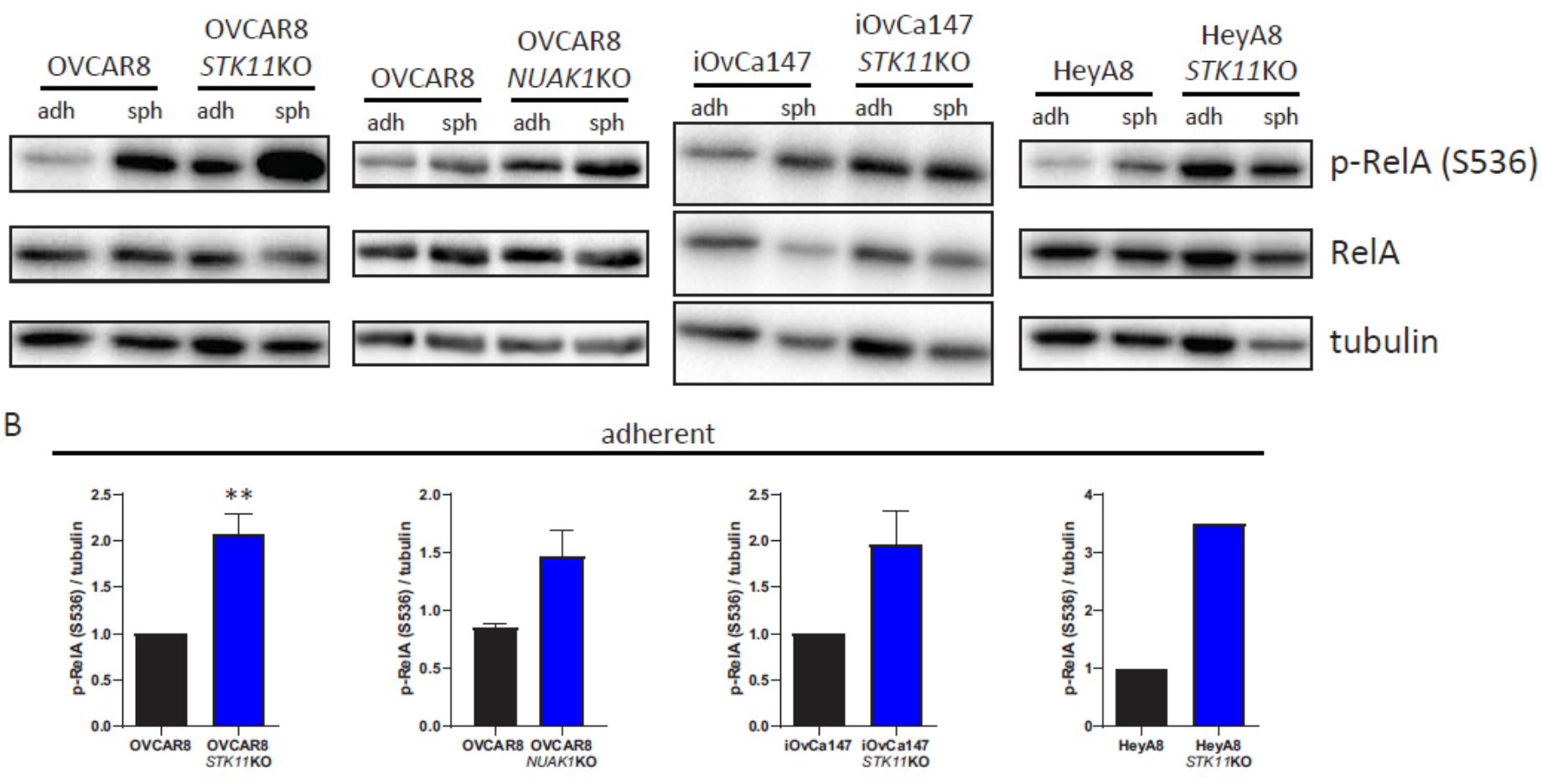

spheroid
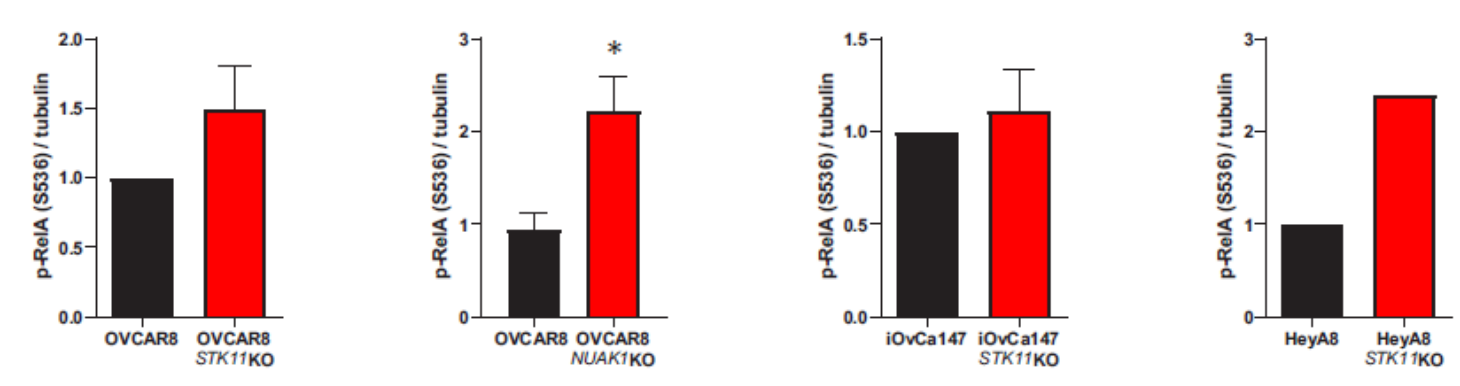

\section{Figure 2}

Increased phosphorylation of RelA at S536 in EOC cell lines lacking LKB1 or NUAK1. (A) Representative immunoblots for p-RelA-S536 in OVCAR8-STK11KO, OVCAR8-NUAK1KO, iOvCa147-STK11KO, and HeyA8STK11KO cell lines and parental lines grown in adherent (adh) and spheroid (sph) culture conditions. Total RelA and tubulin were used as controls. (B) Densitometric analysis of p-RelA-S536 expression with data from adherent culture of knockout cell lines shown in blue and spheroid culture of knockout cell lines are shown in red. Knockout cell lines were compared with parental cell controls (OVCAR8 and iOvCa147) by unpaired, two-tailed Student's t-test ( $\left.p \leq 0.05,{ }^{*} p \leq 0.01 ; n=3\right)$. Error bars indicate standard error of the mean (S.E.M.). 


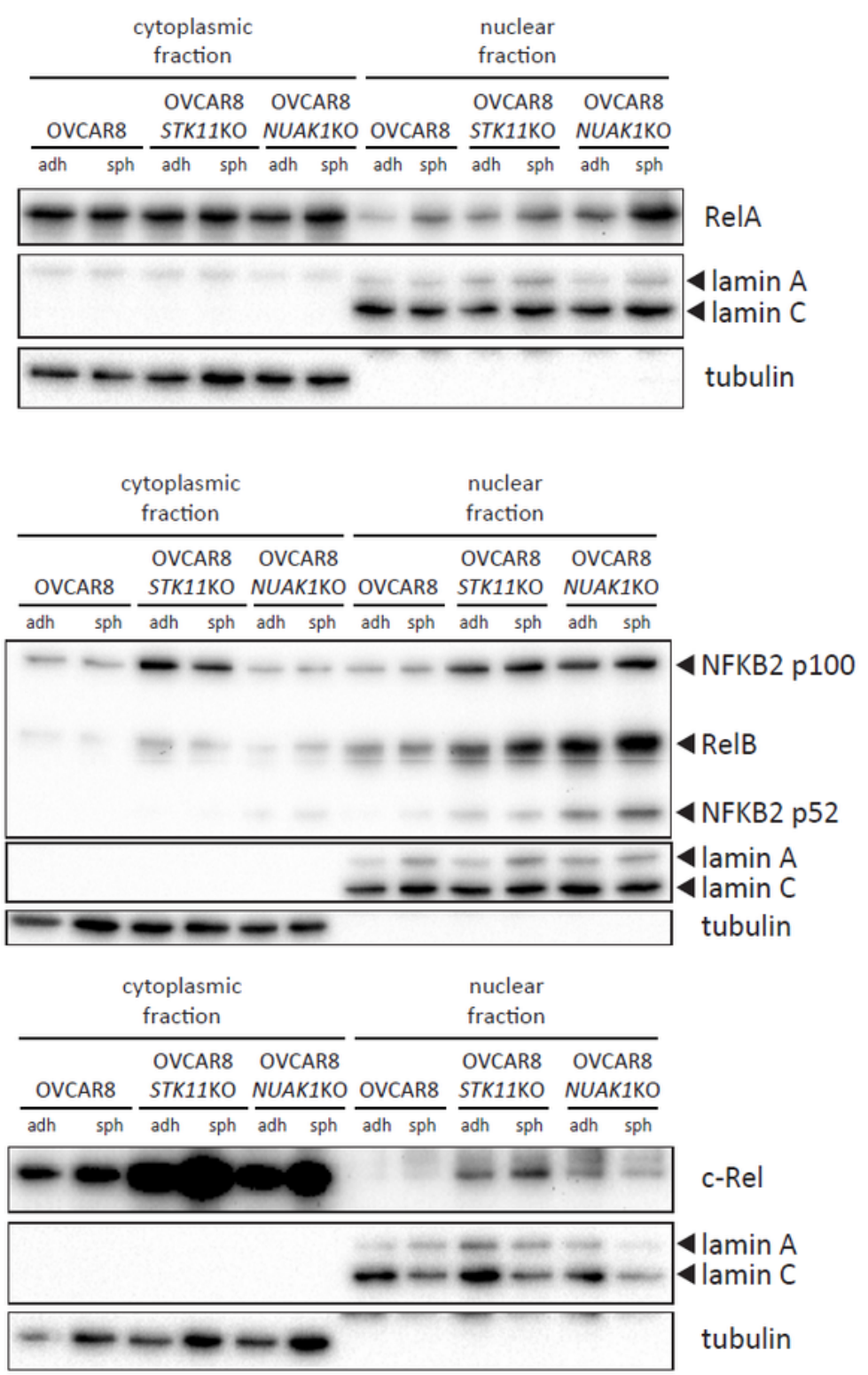

B
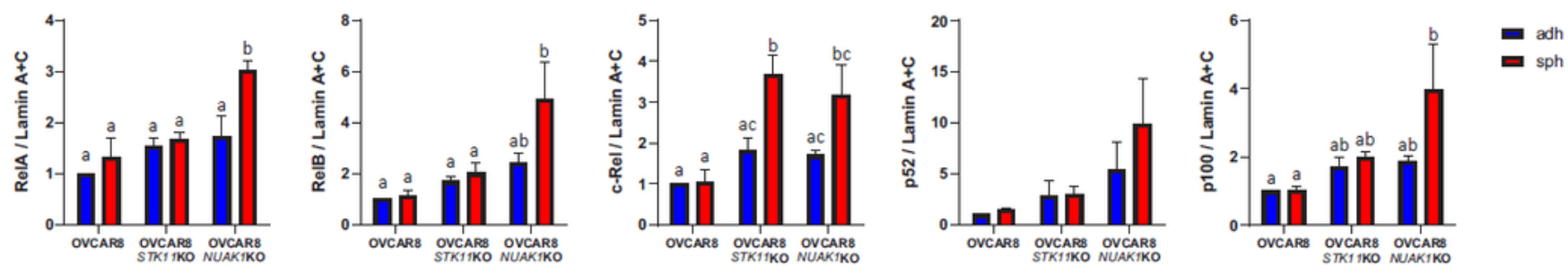

\section{Figure 3}

Increased nuclear abundance of NF-KB family transcription factors in OVCAR8-STK11KO and OVCAR8NUAK1KO spheroid cells. (A) Representative immunoblots of cytoplasmic and nuclear abundance of NFKB transcription factors in OVCAR8, OVCAR8-STK11KO and OVCAR8-NUAK1KO cells grown in adherent (adh) and spheroid (sph) culture conditions. Tubulin and lamin A/C were used as controls for cytoplasmic and nuclear fractions, respectively. (B) Densitometric analysis of NF-kB transcription factor 
protein expression. Data are shown as fold-change relative to parental adherent controls shown in blue (adherent culture) or red (spheroid culture). Statistical analysis was performed by two-way ANOVA and groups were compared by Tukey's multiple comparisons test with alpha set to 0.05 , performing all pairwise comparisons; $n \geq 3$. Letter labels indicate statistically significant differences between groups. Error bars indicate standard error of the mean (S.E.M.).

A
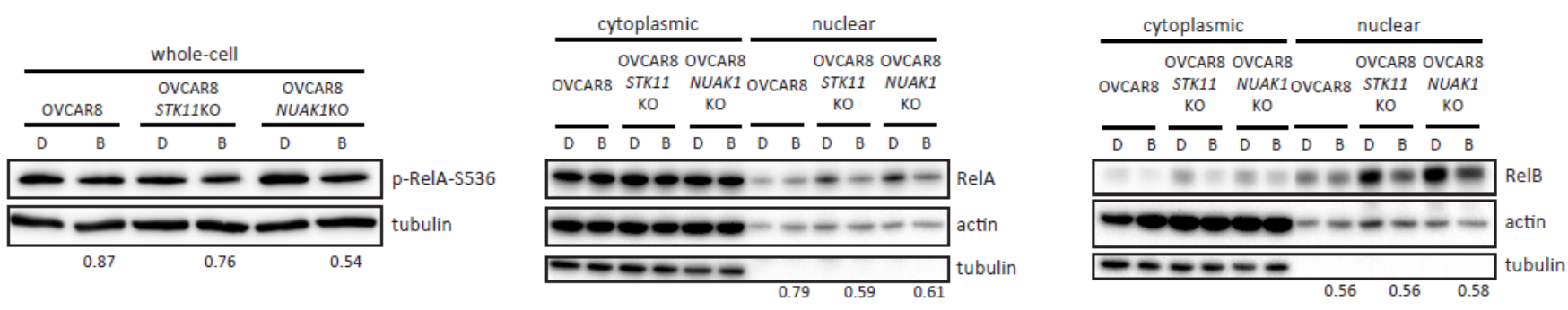

B
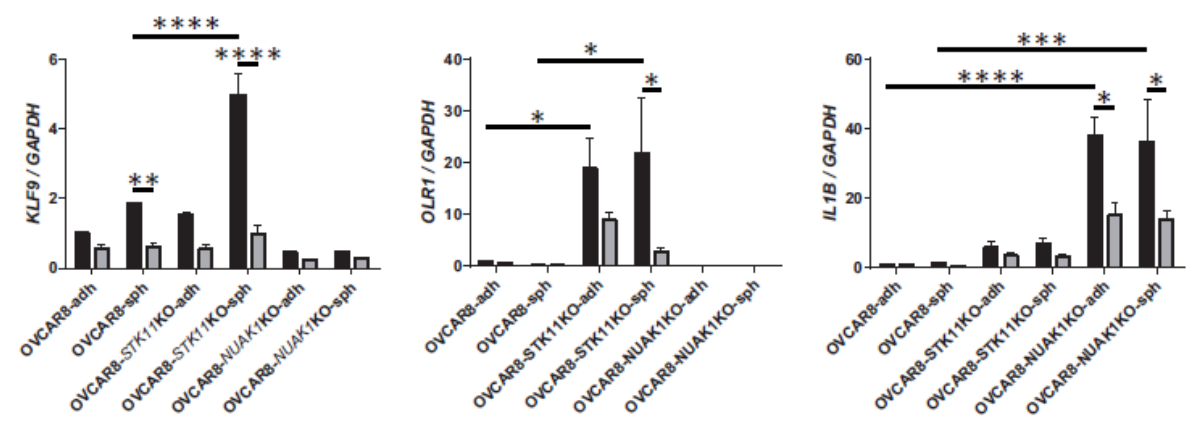

DMSO
BAY 11-7082 (5 uM)

C

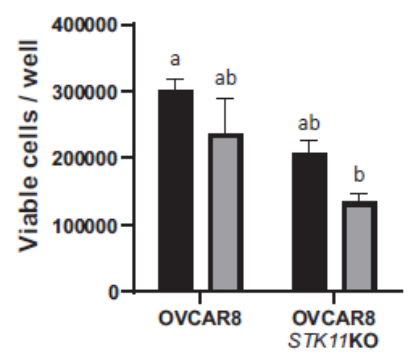

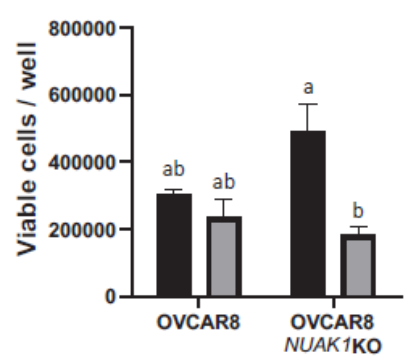

D
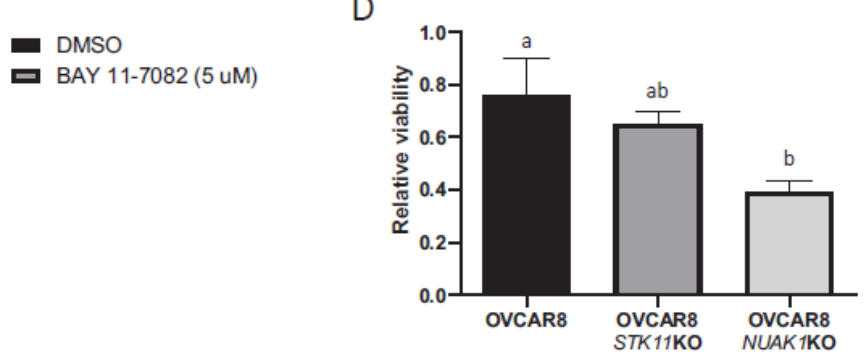

$E$
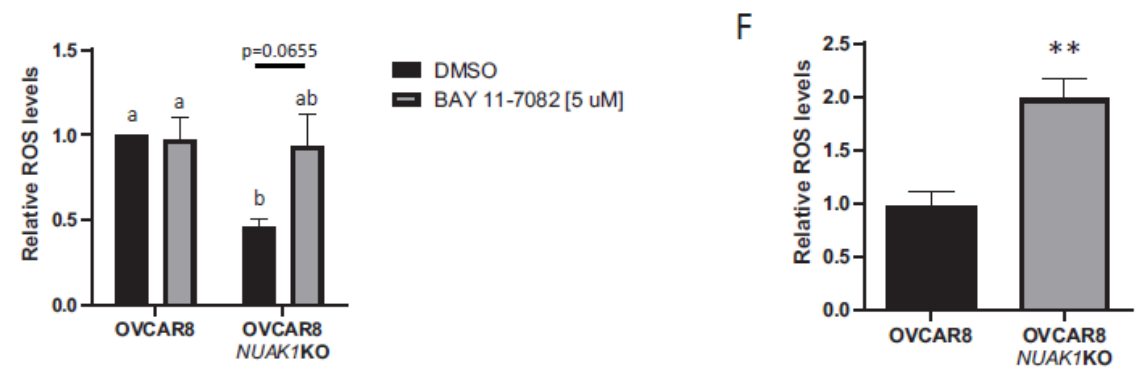

Figure 4

BAY 11-7082 attenuates NF-KB signalling, increases ROS, and decreases viability in OVCAR8-NUAK1KO spheroid cells. (A) Representative immunoblots of spheroid cell lysates showing total abundance of $p$ RelA (S536), and cytoplasmic and nuclear abundance of RelA and RelB in OVCAR8, OVCAR8-STK11KO and OVCAR8-NUAK1KO spheroid cells treated with DMSO (D) or $5 \mu \mathrm{M}$ BAY 11-7082 (B). Values under blot images indicate fold-change relative to DMSO controls. (B) Expression of selected NF-KB target genes in 
OVCAR8, OVCAR8-STK11KO and OVCAR8-NUAK1KO cells treated with DMSO or $5 \mu M$ BAY 11-7082, as measured by RT-qPCR. (C) Viability of OVCAR8, OVCAR8-STK11KO and OVCAR8-NUAK1KO cells cultured as spheroids for five days with DMSO or 5 MM BAY 11-7082. Cell viability was measured using Trypan blue exclusion of dissociated spheroid cells. (D) Relative spheroid cell viability (BAY 11-7082/DMSO) using cell counts shown in (C). (E) ROS levels in OVCAR8 and OVCAR8-NUAK1KO cells cultured as spheroids for seven days with DMSO or $5 \mu \mathrm{M}$ BAY 11-7082. Data are shown as ROS-Glo luminescence values normalized to CyQuant fluorescence values, and fold-change relative to OVCAR8 DMSO-treated control. (F) Relative ROS levels (BAY 11-7082/DMSO) using data shown in (E). (G) Viability of OVCAR8 and OVCAR8-NUAK1KO cells cultured as spheroids for seven days with DMSO or $5 \mu$ M BAY 11-7082. Cell viability was measured using Trypan blue exclusion of dissociated spheroid cells. $(\mathrm{H})$ Relative spheroid cell viability (BAY 11-7082/DMSO) using cell counts shown in (G). For (B), (C), (E), and (G), statistical analysis was performed using two-way ANOVA and groups were compared by Tukey's multiple comparisons test with alpha set to 0.05 , performing all pairwise comparisons. For (D), statistical analysis was performed using one-way ANOVA and groups were compared by Tukey's multiple comparisons test with alpha set to 0.05 , performing all pairwise comparisons. For $(F)$ and $(H)$, knockout cells were compared with parental cells by unpaired, two-tailed Student's t-test. Letter labels indicate statistically significant differences between groups. ( $\mathrm{p} \leq 0.05$, ${ }^{* *} p \leq 0.01$, ${ }^{* *} \mathrm{p} \leq 0.001,{ }^{* * *} \mathrm{p} \leq 0.0001 ; n \geq 3$ ). For all bar graphs, error bars indicate standard error of the mean (S.E.M.).

A
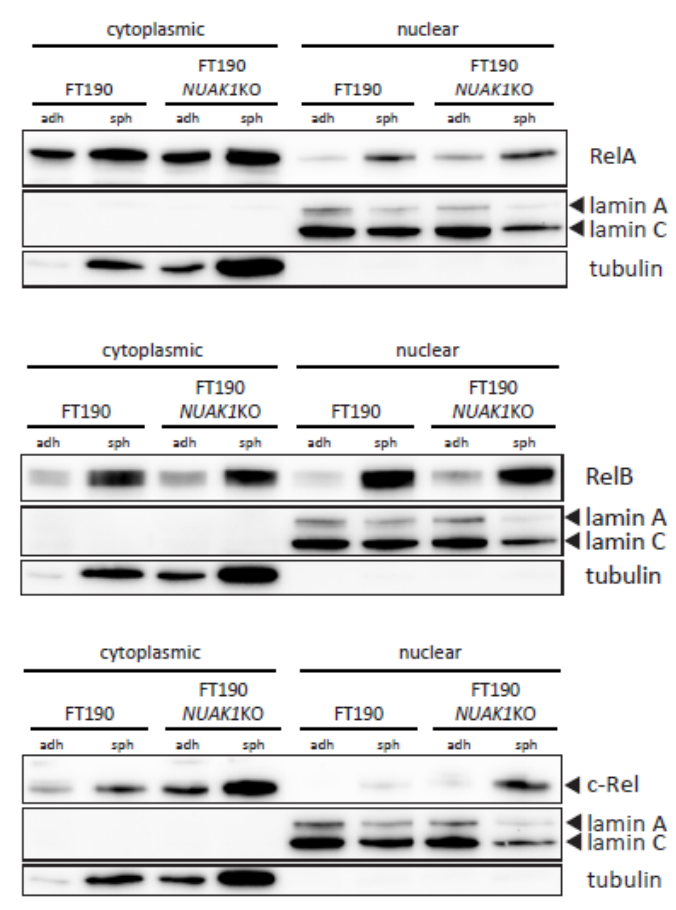

B

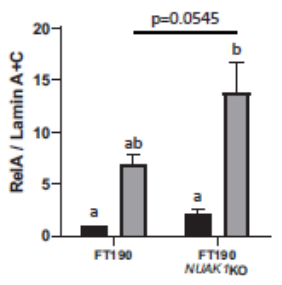

C
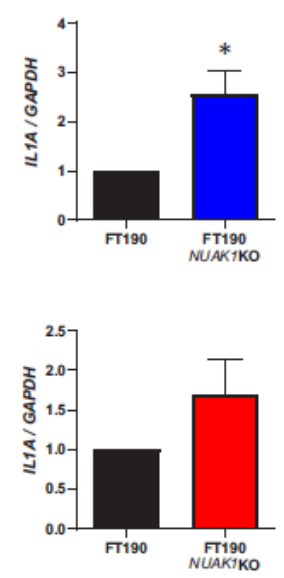
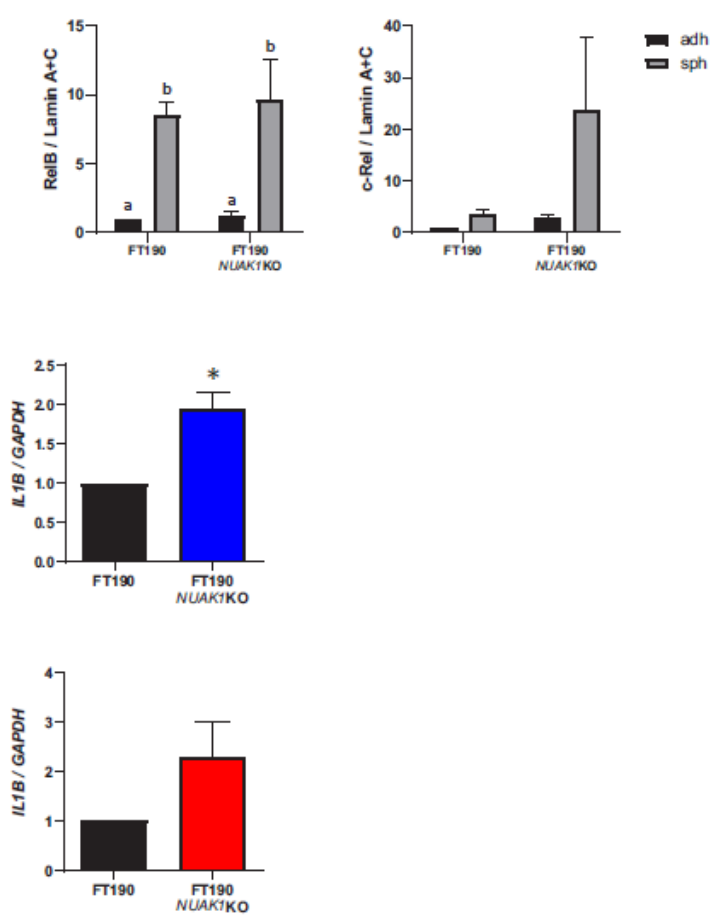

\section{Figure 5}

Nuclear abundance of NF-kB family transcription factors is increased in FT190 spheroid cells. (A) Representative immunoblots of cytoplasmic and nuclear abundance of NF-KB transcription factors in FT190 and FT190-NUAK1KO cells grown in adherent (adh) and spheroid (sph) culture conditions. Tubulin and lamin A/C were used as controls for cytoplasmic and nuclear fractions, respectively. (B) 
Densitometric analysis of NF-KB transcription factor protein expression shown as fold-change relative to parental cells in adherent culture. Statistical analysis was performed using two-way ANOVA followed by Tukey's multiple comparisons test with alpha set to 0.05 , performing all pairwise comparisons; $n=3$. Letter labels indicate statistically significant differences between groups. (C) Fold change in expression of selected NF-KB target genes in FT190-NUAK1KO cells relative to FT190 control cells as determined by RT-qPCR (adherent FT190-NUAK1KO cells shown in blue, and FT190-NUAK1KO spheroid shown in red). Statistical analysis was performed by unpaired, two-tailed Student's t-test (* $p \leq 0.05 ; n=3$ ). For all bar graphs, error bars indicate standard error of the mean (S.E.M.). 


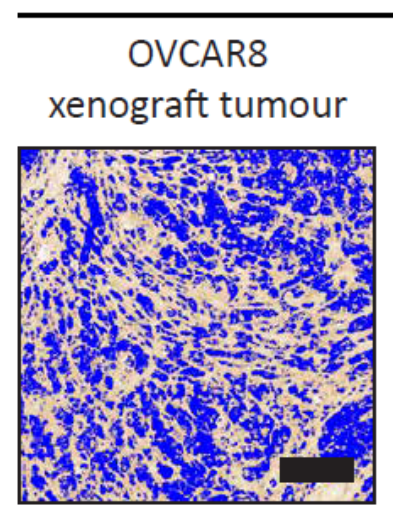

OVCAR8-NUAK1KO

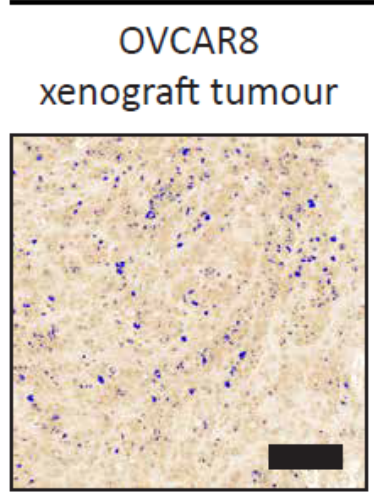

OVCAR8-NUAK1KO

xenograft tumour

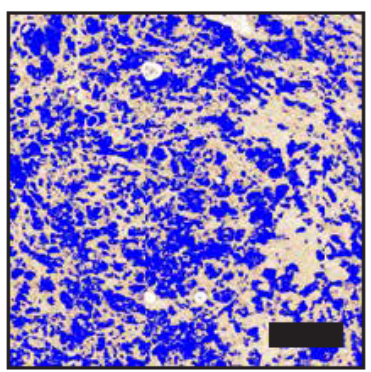

xenograft tumour

B
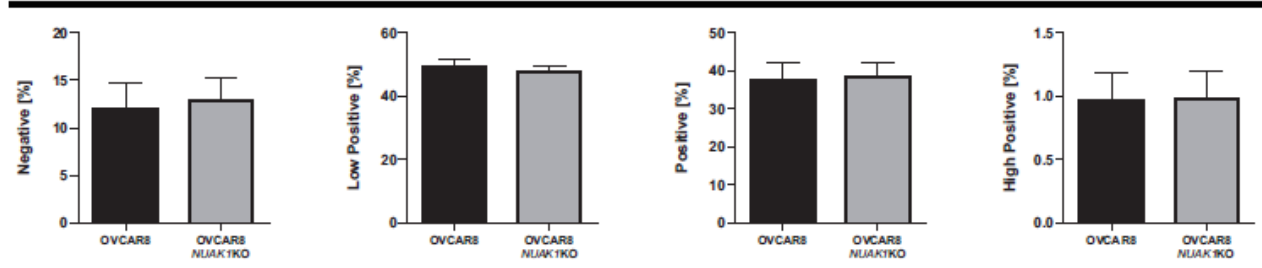

Nuclear
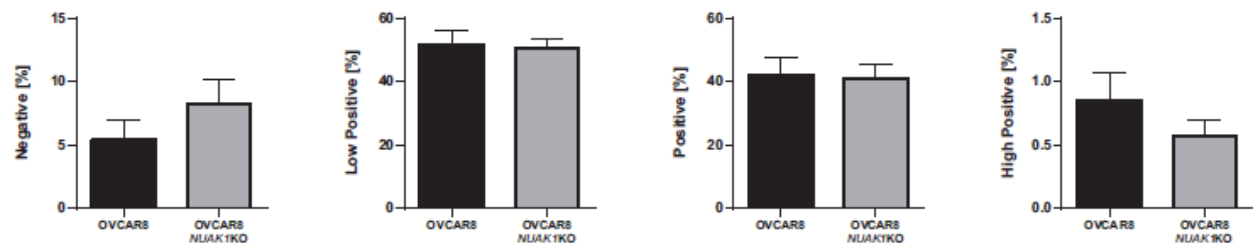

C

Total
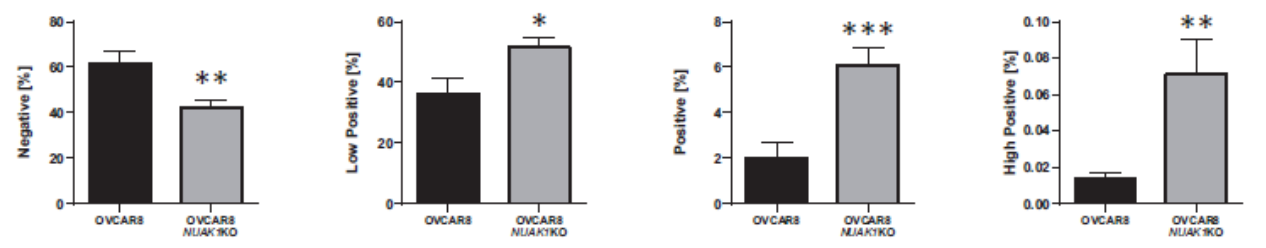

Nuclear
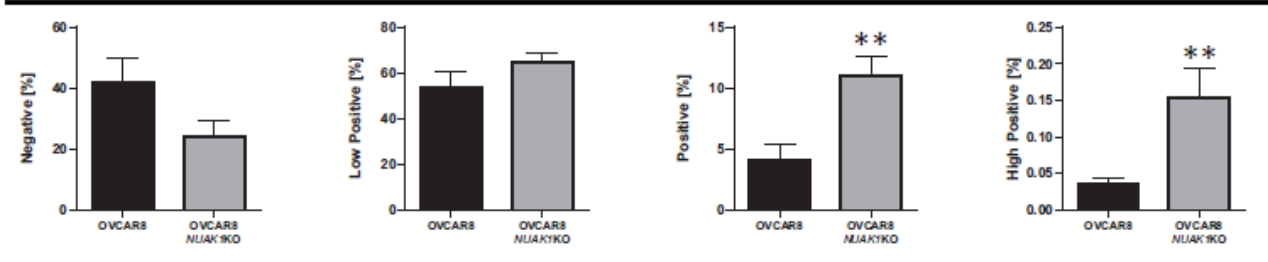

\section{Figure 6}

Enhanced nuclear staining of RelB in OVCAR8-NUAK1KO xenograft tumours. (A) Representative immunohistochemistry DAB channel images for RelA and RelB in xenograft tumours using OVCAR8 and OVCAR8-NUAK1KO cells. Pixels classified as "Positive" and "High Positive" by the IHC Profiler ImageJ plugin are masked blue. Scale bars represent $100 \mu \mathrm{m}$. (B, C) Quantification of DAB staining intensity for RelA (B) and RelB (C) as determined by the IHC Profiler ImageJ plugin. Data are shown as the percentage 
of pixels classified as "Negative", "Low Positive", "Positive”, and "High Positive" for the full image (Total) or nuclear regions as defined by hematoxylin staining (Nuclear). OVCAR8-NUAK1KO were compared with OVCAR8 by unpaired, two-tailed Student's t-test ( ${ }^{*} p \leq 0.05,{ }^{* \star} p \leq 0.01$, $* \star * ~ p \leq 0.001 ; n \geq 10$ tumour samples per group). Error bars indicate standard error of the mean (S.E.M.).

\section{Supplementary Files}

This is a list of supplementary files associated with this preprint. Click to download.

- SupplementaryFigures.pdf

- SupplementaryTableS1.xlsx

- SupplementaryTableS2.xlsx

- UncroppedImmunoblots.pdf 